\title{
On the penalty function and on continuity properties of risk measures
}

\author{
MARCO FRITTELLI EMANUELA ROSAZZA GIANIN
}

March 27, 2010

\begin{abstract}
We discuss two issues about risk measures: we first point out an alternative interpretation of the penalty function in the dual representation of a risk measure; then we analyze the continuity properties of comonotone convex risk measures. In particular, due to the loss of convexity, local and global continuity are no more equivalent and many implications true for convex risk measures do not hold any more.

Dipartimento di Matematica, Università di Milano, Italy.

Dipartimento di Metodi Quantitativi, Università di Milano-Bicocca, Italy. emanuela.rosazza1@unimib.it
\end{abstract} marco.frittelli@unimi.it

\section{Introduction}

This analysis arises from the financial need of weakening the axiom of convexity (or the stronger one of sublinearity) imposed to risk measures (see Delbaen [8], [9], Föllmer and Schied [11], [12] and Frittelli and Rosazza Gianin [18], among many others) and motivated by diversification reasons.

On one hand, indeed, convexity of a real valued map $\pi$ defined on a space of random variables implies that for $\alpha \in[0,1]$

$$
\pi(\alpha X+(1-\alpha) Y) \leq \alpha \pi(X)+(1-\alpha) \pi(Y) \leq \max (\pi(X), \pi(Y)) .
$$

However, to control the risk of the diversified position it is sufficient to assume only quasiconvexity, i.e. $\pi(\alpha X+(1-\alpha) Y) \leq \max (\pi(X), \pi(Y))$. This indeed is one of the motivation behind the recent approach proposed in [5], where the notion of a quasiconvex cash subadditive risk measure was introduced. The dual representation of such risk measures (see Proposition 2) may be written in term of a function $R=R(m, Q)$, which assigns the risk that is headgeable with a level of wealth $m$, once a pricing functional $Q$ is fixed. In the quasiconvex case, this function $R$ replaces the role of the penalty component in the dual representation of a convex risk measure. In the first part of this paper we show that the penalty term of any map $\pi$ can be recovered from the function $R$ by 
computing $\sup _{m \in R}\{m-R(m, Q)\}$, thus proving an alternative interpretation for the penalty function.

On the second hand, insurance premium principles are sometimes assumed to satisfy additivity only for comonotone risks (see Wang et al. [29]). Song and Yan [25] and Heyde et al. [19] proposed therefore to replace convexity with comonotone convexity, that is convexity for comonotone risks. It has been proved by Song and Yan [25] that a risk measure $\rho$ satisfying comonotone convexity (plus some additional assumptions, but without any assumptions on continuity) may be represented as the maximum of the penalized Choquet integrals over a suitable set $\mathcal{M}$ of set functions. In the second part of the paper we show that when continuity from above in 0 is imposed to $\pi(X) \triangleq \rho(-X)$, then in the representation of $\pi$ the set $\mathcal{M}$ may be relaxed with its subset $\mathcal{M}_{\text {ca } 0}$ of set functions that are continuous from above in 0 (see Proposition 16). This result will be applied to some functionals that are law invariant and consistent with different orders. We will consider then different notions of continuity and show that many implications true for convex risk measures fail to hold once convexity is relaxed with comonotone convexity.

\section{Notations and setting}

We consider a lattice $L_{\mathcal{F}} \triangleq L(\Omega, \mathcal{F}, P) \subseteq L^{0}(\Omega, \mathcal{F}, P)$ of $\mathcal{F}$-measurable random variables, on the probability space $(\Omega, \mathcal{F}, P)$, that is endowed with the usual - $P$-a.s. - order relation $\geq$ and we suppose that $L_{\mathcal{F}}$ contains the space $L_{\mathcal{F}}^{\infty}$ of essentially bounded r.v.'s.

The order continuous dual of $\left(L_{\mathcal{F}}, \geq\right)$, denoted by $L_{\mathcal{F}}^{*}=\left(L_{\mathcal{F}}, \geq\right)^{*}$, is a lattice and we assume that it satisfies: $L_{\mathcal{F}}^{*} \hookrightarrow L_{\mathcal{F}}^{1}$. The vector space $L_{\mathcal{F}}^{*}$ is required to be not trivial, so that $\left(L_{\mathcal{F}}, \sigma\left(L_{\mathcal{F}}, L_{\mathcal{F}}^{*}\right)\right)$ is a locally convex TVS.

Many important classes of spaces satisfy these conditions, as for example:

- The $L^{p}$-spaces, $p \in[1, \infty]: L_{\mathcal{F}}=L_{\mathcal{F}}^{p}, L_{\mathcal{F}}^{*}=L_{\mathcal{F}}^{q} \hookrightarrow L_{\mathcal{F}}^{1}$.

- The Orlicz spaces $L^{\Psi}$ for any Young function $\Psi: L_{\mathcal{F}}=L_{\mathcal{F}}^{\Psi}, L_{\mathcal{F}}^{*}=L_{\mathcal{F}}^{\Psi^{*}} \hookrightarrow L_{\mathcal{F}}^{1}$, where $\Psi^{*}$ denotes the conjugate function of $\Psi$.

From now on the dual system $\left(L_{\mathcal{F}}, L_{\mathcal{F}}^{*}\right)$ is fixed.

We also set $\mathcal{P} \triangleq\left\{\frac{d Q}{d P} \in\left(L_{\mathcal{F}}^{1}\right)_{+} \mid Q<<P\right\}$ and, with an abuse of notation, we will write $Q \in \mathcal{P}$ instead of $\frac{d Q}{d P} \in \mathcal{P}$.

Consider the following list of properties on a functional $\pi$ associated to a risk measure $\rho$, that is $\pi(X) \triangleq \rho(-X)$.

Properties of $\pi: L_{\mathcal{F}} \rightarrow \overline{\mathbb{R}} \triangleq \mathbb{R} \cup\{-\infty\} \cup\{\infty\}$.

- monotonicity: $\pi(X) \leq \pi(Y)$ for all $X, Y \in L_{\mathcal{F}}$ such that $X \leq Y$

- convexity: $\pi(\alpha X+(1-\alpha) Y) \leq \alpha \pi(X)+(1-\alpha) \pi(Y)$ for all $X, Y \in L_{\mathcal{F}}$, $\alpha \in[0,1]$

- quasiconvexity: the lower level sets $\mathcal{A}_{\alpha} \triangleq\left\{X \in L_{\mathcal{F}} \mid \pi(X) \leq a\right\}$ are convex $\forall a \in \mathbb{R}$

- additivity: $\pi(X+Y)=\pi(X)+\pi(Y)$ for all $X, Y \in L_{\mathcal{F}}$ 
- subadditivity: $\pi(X+Y) \leq \pi(X)+\pi(Y)$ for all $X, Y \in L_{\mathcal{F}}$

- positive homogeneity: $\pi(\lambda X)=\lambda \pi(X)$ for all $X \in L_{\mathcal{F}}, \lambda \geq 0$

- cash additivity: $\pi(X+a)=\pi(X)+a$ for all $X \in L_{\mathcal{F}}, a \in \mathbb{R}$

- constancy: $\pi(a)=a$ for all $a \in \mathbb{R}$

- law invariance: $\pi(X)=\pi(Y)$ if $X, Y \in L_{\mathcal{F}}$ have the same distribution (notation: $X \sim Y$ )

- lower semicontinuity (lsc): the lower level sets $\mathcal{A}_{a}$ are closed $\forall a \in \mathbb{R}$.

We remind that two random variables $X$ and $Y$ are called comonotone if $\left(X(\omega)-X\left(\omega^{\prime}\right)\right)\left(Y(\omega)-Y\left(\omega^{\prime}\right)\right) \geq 0,(P \times P)$-almost surely. When we add to a property the label "comonotone" it means that the property holds (only) for comonotone r.v.'s.

As mentioned, Section 2 is devoted to the analysis of the penalty term for general maps $\pi: L_{\mathcal{F}} \rightarrow \overline{\mathbb{R}}$. In Section 3.1 a representation of comonotone convex functionals with a continuity property is established. Other notions of continuity and several examples underlining what happens for non-convex functionals are presented in Section 3.2. A sufficient condition for continuity of monotone real-valued functionals is provided in Section 3.3.

\section{On the penalty function of risk measures}

In this section we consider maps $\pi: L_{\mathcal{F}} \rightarrow \overline{\mathbb{R}} \triangleq \mathbb{R} \cup\{-\infty\} \cup\{\infty\}$. We first recall that the convex conjugate of a map $\pi: L_{\mathcal{F}} \rightarrow \overline{\mathbb{R}}$ is the convex $\left(L_{\mathcal{F}}^{*}, L_{\mathcal{F}}\right)$-lsc function $\pi^{*}: L_{\mathcal{F}}^{*} \rightarrow \overline{\mathbb{R}}$ defined by

$$
\pi^{*}(Z)=\sup _{\xi \in L_{\mathcal{F}}}\{E[Z \xi]-\pi(\xi)\} .
$$

The dual representation of "quasiconvex risk measures" (see [5] in the static case and [16] in a dynamic setting) is written in terms of the following functions of two variables.

Definition 1 Let $\pi: L_{\mathcal{F}} \rightarrow \overline{\mathbb{R}}$ and set for $Z \in L_{\mathcal{F}}^{*}$ and $m \in \mathbb{R}$

$$
\begin{aligned}
& R(m, Z) \triangleq \inf _{\xi \in L_{\mathcal{F}}}\{\pi(\xi) \mid E[Z \xi]=m\}, \\
& \mathcal{R}(m, Z) \triangleq \inf _{\xi \in L_{\mathcal{F}}}\{\pi(\xi) \mid E[Z \xi] \geq m\},
\end{aligned}
$$

and $R(m, Q) \triangleq R\left(m, \frac{d Q}{d P}\right)$, if $Q \in \mathcal{P}$.

Interpretation of $R(m, Q)$

For a given level of wealth $m$ and for a given "pricing functional" $Q, R(m, Q)$ assigns the "value" or "risk" - depending on the meaning of the map $\pi$, either as value or as risk - that is "attainable" or "hedgeable" from $m$. 
Notice that if $Z \in\left(L_{\mathcal{F}}^{*}\right)_{+}$with $E[Z]>0$ and $\frac{d Q}{d P} \triangleq \frac{Z}{E[Z]}$ then

$$
R(E[Z X], Z)=R\left(\frac{E[Z X]}{E[Z]}, \frac{Z}{E[Z]}\right)=R\left(E_{Q}[X], Q\right)
$$

The following proposition reassumes some of the results in [5] and [16], which are essentially based on those proved in [28], on the dual representation of quasiconvex functions. We recall that $\pi: L_{\mathcal{F}} \rightarrow \overline{\mathbb{R}}$ is quasiconvex if and only if $\pi(\alpha X+(1-\alpha) Y) \leq \max (\pi(X), \pi(Y))$, for all $X, Y \in L_{\mathcal{F}}, \alpha \in[0,1]$.

Proposition 2 (i) If $\pi: L_{\mathcal{F}} \rightarrow \overline{\mathbb{R}}$ is quasiconvex and $\left(L_{\mathcal{F}}, L_{\mathcal{F}}^{*}\right)$-lsc then

$$
\pi(X)=\sup _{Z \in L_{\mathcal{F}}^{*}} \mathcal{R}(E[Z X], Z)
$$

(ii) If in addition $\pi$ is monotone increasing then

$$
\pi(X)=\sup _{Z \in L_{\mathcal{F}}^{*} \cap \mathcal{P}} R(E[Z X], Z)
$$

(iii) If in addition $\pi$ is cash additive then

$$
R(m, Q)=m-\pi^{*}(Q)
$$

and therefore, from (2) and (3):

$$
\pi(X)=\sup _{Q \in L_{\mathcal{F}}^{*} \cap \mathcal{P}}\left\{E_{Q}[X]-\pi^{*}(Q)\right\}
$$

Remark 3 The formula (4) can be deduced from the equality $\pi=\pi^{* *}$, which holds for any convex lsc proper function - as stated by the Fenchel Moreau theorem - and using monotonicity and cash additivity (see [18]). The fact that it is possible to obtain (4) from quasiconvexity is not a surprise since quasiconvexity and cash additivity imply convexity. This last sentence is well known (see for example [5]) and can be proved in a direct way as follows. By quasiconvexity, the lower level set $\mathcal{A}_{0}$ is convex and, by cash additivity, $(X-\pi(X)) \in \mathcal{A}_{0}$ for any $X \in L_{\mathcal{F}}$. Therefore, $\{\lambda(X-\pi(X))+(1-\lambda)(Y-\pi(Y))\} \in \mathcal{A}_{0}$, for any $X, Y \in L_{\mathcal{F}}$ and $\lambda \in[0,1]$ and:

$$
\begin{aligned}
0 & \geq \pi\{\lambda(X-\pi(X))+(1-\lambda)(Y-\pi(Y))\} \\
& =\pi(\lambda X+(1-\lambda) Y)-\lambda \pi(X)-(1-\lambda) \pi(Y)
\end{aligned}
$$

by cash additivity.

Remark 4 Setting $\rho(X)=\pi(-X)$ we obtain from (4) the well known (see [11] and [18]) representation of a convex risk measures

$$
\rho(X)=\sup _{Q \in L_{\mathcal{F}}^{*} \cap \mathcal{P}}\left\{E_{Q}[-X]-\rho^{*}(-Q)\right\}
$$


Example 5 For a given utility function $u$, that is a strictly increasing concave function $u: \mathbb{R} \rightarrow \mathbb{R}$, consider the certainty equivalent operator $\pi^{u}(\cdot)=$ $u^{-1}\left(E_{P}[u(\cdot)]\right)$. Notice that $\pi^{u}$ is a quasiconcave map, that in general is not concave, and we assume that $\pi^{u}: L_{\mathcal{F}} \rightarrow \overline{\mathbb{R}}$ is well defined (this is the case, for example, if $L_{\mathcal{F}}$ is the Orlicz space associated to the utility function u, see [17] for details). In the presence of a financial market, the no arbitrage principle generates the set of equivalent martingale (or pricing) measures. For an assigned "pricing" measure $Q \in L_{\mathcal{F}}^{*} \cap \mathcal{P}$ consider the price $E_{Q}[X]$ of the claim $X$. As in [2] and [3], we compare two components: the artificial linear pricing operator $E_{Q}[\cdot]$ and the subjective valuation $\pi^{u}(\cdot)$ based on the preference relation $\succeq$ associated to $u$ (and $P$ ):

$$
E_{Q}[X]-\pi^{u}(X)=E_{Q}[X]-u^{-1}\left(E_{P}[u(X)]\right) .
$$

The maximum of this difference, for a fixed level $m$, is

$$
\begin{aligned}
\sup _{\xi \in L_{\mathcal{F}}: E_{Q}[\xi]=m}\left\{E_{Q}[\xi]-u^{-1}\left(E_{P}[u(\xi)]\right)\right\} & =\sup _{\xi \in L_{\mathcal{F}}: E_{Q}[\xi]=m}\left\{m-\pi^{u}(\xi)\right\} \\
& =m-R(m, Q) .
\end{aligned}
$$

In general this difference will depend on the level of wealth $m$, as well as on $Q$, and its maximum, with respect to all $m \in \mathbb{R}$

$$
\pi^{\circledast}(Q) \triangleq \sup _{m \in \mathbb{R}}\{m-R(m, Q)\}=\sup _{m \in \mathbb{R}} \sup _{\left\{\xi \in L_{\mathcal{F}}: E_{Q}[\xi]=m\right\}}\left\{E_{Q}[\xi]-u^{-1}\left(E_{P}[u(\xi)]\right)\right\},
$$

gives the "distance" between the certainty equivalent operator $\pi^{u}$ and the expectation operator w.r.to the fixed $Q$.

This example suggests to define the following penalty function $\pi^{\circledast}$ of $\pi$.

Definition 6 Let $\pi: L_{\mathcal{F}} \rightarrow \overline{\mathbb{R}}, Z \in L_{\mathcal{F}}^{*}$ and set:

$$
\begin{aligned}
R^{\pi}(m, Z) & \triangleq m-R(m, Z), \\
\pi^{\circledast}(Z) & \triangleq \sup _{m \in \mathbb{R}}\{m-R(m, Z)\}=\sup _{m \in \mathbb{R}}\left\{R^{\pi}(m, Z)\right\} .
\end{aligned}
$$

Notice that when $Z=0, R(m, 0)$ and $\mathcal{R}(m, 0)$ are equal to $+\infty$ for $m \neq 0$ and $R(0,0)=\mathcal{R}(0,0)=\inf _{\xi \in L_{\mathcal{F}}}\{\pi(\xi)\}, \pi^{\circledast}(0)=\pi^{*}(0)=-R(0,0)$.

Let us also consider the symmetric notations and state the corresponding dual representation in the concave upper semicontinuous (usc) case.

Definition 7 Let $\pi: L_{\mathcal{F}} \rightarrow \overline{\mathbb{R}}$ and set for $Z \in L_{\mathcal{F}}^{*}$ and $m \in \mathbb{R}$

$$
\begin{gathered}
r(m, Z) \triangleq \sup _{\xi \in L_{\mathcal{F}}}\{\pi(\xi) \mid E[Z \xi]=m\}, \\
\pi_{\circledast}(Z) \triangleq \inf _{m \in \mathbb{R}}\{m-r(m, Z)\}
\end{gathered}
$$

and let $\pi_{*}: L_{\mathcal{F}}^{*} \rightarrow \overline{\mathbb{R}}$ be the concave conjugate of $\pi$ :

$$
\pi_{*}(Z)=\inf _{\xi \in L_{\mathcal{F}}}\{E[Z \xi]-\pi(\xi)\} .
$$


Proposition 8 (i) If $\pi: L_{\mathcal{F}} \rightarrow \overline{\mathbb{R}}$ is quasiconcave, monotone increasing and $\left(L_{\mathcal{F}}, L_{\mathcal{F}}^{*}\right)-$ usc then

$$
\pi(X)=\inf _{Q \in L_{\mathcal{F}}^{*} \cap \mathcal{P}} r\left(E_{Q}[X], Q\right) .
$$

(ii) If in addition $\pi$ is cash additive then

$$
\begin{gathered}
r(m, Q)=m-\pi_{*}(Q), \\
\pi(X)=\inf _{Q \in L_{\mathcal{F}}^{*} \cap \mathcal{P}}\left\{E_{Q}[X]-\pi_{*}(Q)\right\} .
\end{gathered}
$$

In the next proposition we show that for any map $\pi$ (in particular we do not assume that $\pi$ is quasiconvex, monotone or cash additive) $\pi^{\circledast}$ coincides with the convex conjugate $\pi^{*}$; moreover, when $\pi$ is cash additive, then $m-R(m, Z)$ does not depend on $m$, so that $\pi^{\circledast}(Q)=-R(0, Q)=R^{\pi}(m, Q)$ for any $m$.

Proposition 9 Let $\pi: L_{\mathcal{F}} \rightarrow \overline{\mathbb{R}}, Q \in L_{\mathcal{F}}^{*} \cap \mathcal{P}$ and $m \in \mathbb{R}$.

1.

$$
\pi_{*}=\pi_{\circledast} \leq \pi^{\circledast}=\pi^{*} .
$$

2. If $\pi$ is cash additive then $R(\cdot, Q)$ and $r(\cdot, Q)$ are affine and

$$
\begin{gathered}
R(m, Q)=m-\pi^{*}(Q), r(m, Q)=m-\pi_{*}(Q) \\
\pi_{*}(Q)=\pi_{\circledast}(Q)=-r(0, Q) \leq-R(0, Q)=\pi^{\circledast}(Q)=\pi^{*}(Q) .
\end{gathered}
$$

Proof. The statements regarding $R(\cdot, Q), \pi^{\circledast}$ and $\pi^{*}$ are proved in Lemma 13 , where few additional items are also showed. The statements concerning $r(\cdot, Q), \pi_{\circledast}$ and $\pi_{*}$ can be proved in a similar way. The two inequalities are simple consequences of the definitions.

Example 10 In the case of the exponential utility function it is known (see [14], Proposition 3.2) that

$$
\frac{1}{a} H(Q, P)=\sup _{\xi \in L_{\mathcal{F}}: E_{\mathcal{Q}}[\xi]=0} u_{a}^{-1}\left(E_{P}\left[u_{a}(\xi)\right]\right),
$$

where: $u_{a}(x)=-\frac{1}{a} e^{-a x}, a>0, H(Q, P)=E\left[\frac{d Q}{d P} \log \left(\frac{d Q}{d P}\right)\right]$ is the relative entropy and $L_{\mathcal{F}}=L_{\mathcal{F}}^{\infty}$.

Here, $\pi^{a}(X) \triangleq u_{a}^{-1}\left(E_{P}\left[u_{a}(X)\right]\right)=-\frac{1}{a} \ln \left(E\left[e^{-a X}\right]\right)$ is cash additive and therefore from item (2) of Proposition 9 and from the definition of $r$ in equation (5) we get

$$
-\pi_{*}^{a}(Q)=-\pi_{\circledast}^{a}(Q)=r^{a}(0, Q) \triangleq \sup _{\xi \in L_{\mathcal{F}}: E_{Q}[\xi]=0} u_{a}^{-1}\left(E_{P}\left[u_{a}(\xi)\right]\right)=\frac{1}{a} H(Q, P),
$$

and we recover the well known penalty function of the entropic risk measure $\rho^{a}$ defined by

$$
\rho^{a}(X) \triangleq-\pi^{a}(X)=\frac{1}{a} \ln \left(E\left[e^{-a X}\right]\right) .
$$


Notice indeed that, since $\pi^{a}$ is quasiconcave, $\left(L_{\mathcal{F}}^{\infty}, L_{\mathcal{F}}^{1}\right)$-usc, monotone increasing and cash additive, the equation (6) and $\pi_{*}^{a}(Q)=-\frac{1}{a} H(Q, P)$ imply

$\rho^{a}(X)=-\pi^{a}(X)=-\inf _{Q \in L_{\mathcal{F}}^{1} \cap \mathcal{P}}\left\{E_{Q}[X]-\pi_{*}^{a}(Q)\right\}=\sup _{Q<<P}\left\{E_{Q}[-X]-\frac{1}{a} H(Q, P)\right\}$.

But the circumstance that $m-r(m, Z)$ does not depend on $m$ is specific of the selection of the exponential utility, as in this example; in general to recover the penalty function $\pi_{*}=\pi_{\circledast}$ a further optimization with respect to $m$ is needed.

Proposition 11 Let $\pi: L_{\mathcal{F}} \rightarrow \overline{\mathbb{R}}, Z \in L_{\mathcal{F}}^{*}$. Then

$$
\pi^{\circledast}(Z)=\sup _{X \in L_{\mathcal{F}}} R^{\pi}(E[Z X], Z)=\pi^{*}(Z) .
$$

For $X \in L_{\mathcal{F}}$, the duality relation

$$
\pi(X)=\sup _{Z \in\left(L_{\mathcal{F}}^{*}\right)_{+}}\left\{E[Z X]-R^{\pi}(E[Z X], Z)\right\}
$$

holds true under the conditions that $\pi$ is quasiconvex, $\left(L_{\mathcal{F}}, L_{\mathcal{F}}^{*}\right)$-lower semicontinuous and monotone increasing.

Proof. Equation (7) holds true if $Z=0$, and so we now suppose $Z \neq 0$. Since $L_{\mathcal{F}}^{\infty} \subseteq L_{\mathcal{F}}$, the range of $X \rightarrow E[Z X]$ is $\mathbb{R}$ and so:

$$
\pi^{\circledast}(Z) \triangleq \sup _{m \in \mathbb{R}}\left\{R^{\pi}(m, Z)\right\}=\sup _{X \in L_{\mathcal{F}}} R^{\pi}(E[Z X], Z) .
$$

Equation (8) is a reformulation of equation (2).

Remark 12 Suppose that $\pi$ is a convex lsc proper monotone increasing function. From the Fenchel Moreau theorem $\pi^{* *}=\pi$ and from (8) we get:

$$
\sup _{Z \in\left(L_{\mathcal{F}}^{*}\right)_{+}}\left\{E[Z X]-\pi^{*}(Z)\right\}=\pi(X)=\sup _{Z \in\left(L_{\mathcal{F}}^{*}\right)_{+}}\left\{E[Z X]-R^{\pi}(E[Z X], Z)\right\} .
$$

In general $\pi^{*}(Z) \neq R^{\pi}(E[Z X], Z)$, while if $\pi$ is cash additive then $\pi^{*}(Z)=$ $R^{\pi}(m, Z)$, for any $m$.

Lemma 13 Let $\pi: L_{\mathcal{F}} \rightarrow \overline{\mathbb{R}}, Z \in L_{\mathcal{F}}^{*}$ and $m \in \mathbb{R}$.

1.

$$
\begin{gathered}
R(m, Z) \geq \mathcal{R}(m, Z) \geq m-\pi^{*}(Z), \\
\sup _{m \in \mathbb{R}}\{m-R(m, Z)\}=\sup _{m \in \mathbb{R}}\{m-\mathcal{R}(m, Z)\}, \\
\pi^{\circledast}(Z)=\pi^{*}(Z) .
\end{gathered}
$$


2. Suppose that $\pi$ is cash additive. For all $E[Z] \neq 0$ and $m, c \in \mathbb{R}$

$$
R(c+m, Z)=R(c, Z)+\frac{m}{E[Z]}
$$

and:

$$
R\left(m, \frac{Z}{E[Z]}\right)=m-\pi^{*}\left(\frac{Z}{E[Z]}\right)=m+R(0, Z)
$$

3. Suppose that $\pi$ is cash sub-additive, i.e. $\pi(X+m) \leq \pi(X)+m$, for all $m \geq 0$ and $X \in L_{\mathcal{F}}$. For all $E[Z]>0, c \in \mathbb{R}$ and $m \in \mathbb{R}_{+}$

$$
\mathcal{R}(c+m, Z) \leq \mathcal{R}(c, Z)+\frac{m}{E[Z]}, R(c+m, Z) \leq R(c, Z)+\frac{m}{E[Z]} .
$$

\section{Proof.}

1. We may assume that $Z \neq 0$, since for $Z=0$ the statements in item 1 are obvious. For all $\xi \in L_{\mathcal{F}}$ we have: $\pi^{*}(Z) \triangleq \sup _{\xi \in L_{\mathcal{F}}}\{E[Z \xi]-\pi(\xi)\} \geq$ $E[Z \xi]-\pi(\xi)$. Hence: $m-\pi^{*}(Z) \leq m-E[Z \xi]+\pi(\xi) \leq \pi(\xi)$ for all $\xi \in L_{\mathcal{F}}$ s.t. $E[Z \xi] \geq m$. Therefore: $m-\pi^{*}(Z) \leq \inf _{\xi \in L_{\mathcal{F}}}\{\pi(\xi) \mid E[Z \xi] \geq m\}=$ $\mathcal{R}(m, Z) \leq R(m, Z)$. Then $m-\mathcal{R}(m, Z) \leq \pi^{*}(Z)$ and

$$
\pi^{\circledast}(Z)=\sup _{m \in \mathbb{R}}\{m-R(m, Z)\} \leq \sup _{m \in \mathbb{R}}\{m-\mathcal{R}(m, Z)\} \leq \pi^{*}(Z) .
$$

Now let $X \in L_{\mathcal{F}}$. Notice that (since $Z \neq 0$ ) the range of $X \rightarrow E[Z X]$ is $\mathbb{R}$ and so:

$$
\pi^{\circledast}(Z) \triangleq \sup _{m \in \mathbb{R}}\left\{R^{\pi}(m, Z)\right\}=\sup _{X \in L_{\mathcal{F}}} R^{\pi}(E[Z X], Z) .
$$

Since $\inf _{\xi \in L_{\mathcal{F}}}\{\pi(\xi) \mid E[Z \xi]=E[Z X]\} \leq \pi(X)$ we have:

$R^{\pi}(E[Z X], Z)=E[Z X]-\inf _{\xi \in L_{\mathcal{F}}}\{\pi(\xi) \mid E[Z \xi]=E[Z X]\} \geq E[Z X]-\pi(X)$,

which implies

$$
\sup _{X \in L_{\mathcal{F}}} R^{\pi}(E[Z X], Z) \geq \sup _{X \in L_{\mathcal{F}}}\{E[Z X]-\pi(X)\}=\pi^{*}(Z)
$$

and taking into consideration (10) and (9) we deduce

$$
\pi^{\circledast}(Z)=\sup _{X \in L_{\mathcal{F}}} R^{\pi}(E[Z X], Z) \geq \pi^{*}(Z) \geq \sup _{m \in \mathbb{R}}\{m-\mathcal{R}(m, Z)\} \geq \pi^{\circledast}(Z)
$$

which concludes the proof of item 1 .

2. Since $\pi$ is cash additive, it is easy to check that for $m, c \in \mathbb{R}$ and $E[Z] \neq 0$

$$
R(c+m, Z)=R(c, Z)+\frac{m}{E[Z]},
$$


and therefore, letting $c=0$,

$$
R\left(m, \frac{Z}{E[Z]}\right)=R\left(0, \frac{Z}{E[Z]}\right)+m=R(0, Z)+m .
$$

From $\pi^{*}=\pi^{\circledast}$ we then deduce:

$$
\pi^{*}\left(\frac{Z}{E[Z]}\right)=\pi^{\circledast}\left(\frac{Z}{E[Z]}\right) \triangleq \sup _{m \in \mathbb{R}}\left\{m-R\left(m, \frac{Z}{E[Z]}\right)\right\}=-R(0, Z) .
$$

and therefore

$$
R\left(m, \frac{Z}{E[Z]}\right)=m+R(0, Z)=m-\pi^{*}\left(\frac{Z}{E[Z]}\right) .
$$

3. We prove only the first inequality, since the same argument can be used to prove the second one. Let $\eta \triangleq \xi-\frac{m}{E[Z]}$, than we have

$$
\begin{aligned}
& \mathcal{R}(c+m, Z) \triangleq \inf _{\xi \in L_{\mathcal{F}}}\{\pi(\xi) \mid E[Z \xi] \geq c+m\} \\
= & \inf _{\xi \in L_{\mathcal{F}}}\left\{\pi(\xi) \mid E\left[Z\left(\xi-\frac{m}{E[Z]}\right)\right] \geq c\right\} \\
= & \inf _{\eta \in L_{\mathcal{F}}}\left\{\pi\left(\eta+\frac{m}{E[Z]}\right) \mid E[Z \eta] \geq c\right\} .
\end{aligned}
$$

Notice that $\frac{m}{E[Z]} \geq 0$ then, from cash sub-additivity of $\pi$, we obtain

$$
\mathcal{R}(c+m, Z) \leq \inf _{\eta \in L_{\mathcal{F}}}\{\pi(\eta) \mid E[Z \eta] \geq c\}+\frac{m}{E[Z]} \triangleq \mathcal{R}(c, Z)+\frac{m}{E[Z]}
$$

\section{On continuity properties of risk measures}

It is well known that a convex function bounded above on a neighborhood of a point is continuous at that point. This in particular implies that for convex functions local and global continuity are equivalent (see, for instance, Aliprantis and Border [1]).

In this section we analyze the continuity properties of comonotone convex risk measure, where the above equivalence (as well as many other implications true for convex risk measures) does not hold true any more, due to the loss of convexity. In the last subsection we also provide a simple criterium for continuity for monotone real-valued maps.

In the sequel of the paper we assume $L_{\mathcal{F}}=L_{\mathcal{F}}^{\infty}$.

There are some trivial implications among some of the properties of $\pi$ listed in the introduction. Obviously convexity implies comonotone convexity; furthermore, comonotone additivity and constancy imply cash additivity. Indeed, 
it is straightforward to check that a constant random variable $a$ is comonotone with any random variable $X \in L_{\mathcal{F}}$.

Subadditivity, positive homogeneity and $\pi( \pm 1)= \pm 1$ imply cash additivity (see [15]). More generally, it is easy to check that comonotone subadditivity, positive homogeneity and $\pi( \pm 1)= \pm 1$ imply cash additivity.

In order to show the dual representation of comonotone risk measures we still need to introduce some properties (see for reference Denneberg [10], Aliprantis and Border [1], among many others) of set functions $\mu: \mathcal{F} \rightarrow[0,+\infty]$. We assume that each set function $\mu$ satisfies $\mu(\varnothing)=0$.

\section{Properties on $\mu$}

- monotone: if $A, B \in \mathcal{F}, A \subseteq B$, then $\mu(A) \leq \mu(B)$

- finite: if $\mu(A)<+\infty$ for any $A \in \mathcal{F}$. In particular, $\mu$ is said to be normalized if $\mu(\Omega)=1$

- submodular (or 2-alternating): if $A, B \in \mathcal{F}(A \cup B, A \cap B \in \mathcal{F})$, then $\mu(A \cup B)+\mu(A \cap B) \leq \mu(A)+\mu(B)$

- continuous from below: if $\left(A_{n}\right)_{n>0} \subseteq \mathcal{F}, A_{n} \subseteq A_{n+1}$ for any $n \geq 0$ (and $\left.A=\cup_{n=0}^{\infty} A_{n} \in \mathcal{F}\right)$, then $\lim _{n} \mu\left(A_{n}\right)=\mu(A)$

- continuous from above: if $\left(B_{n}\right)_{n>0} \subseteq \mathcal{F}, B_{n} \supseteq B_{n+1}$ for any $n \geq 0$ (and $\left.B=\cap_{n=0}^{\infty} B_{n} \in \mathcal{F}\right)$, then $\lim _{n} \mu\left(B_{n}\right)=\mu(B)$

- absolutely continuous with respect to $P(\mu \ll P)$ : if $\mu$ is a normalized monotone set function such that: for any $A, B \in \mathcal{F}$ with $P(A \triangle B)=0$ it holds that $\mu(A)=\mu(B)$.

Consider, for instance, an increasing function $f:[0,1] \rightarrow[0,1]$ satisfying $f(0)=0$ and $f(1)=1$. Such a function $f$ is called distortion and the (monotone and normalized) set function $\mu \triangleq f \circ P$ associated to $f$ is called distorted probability. Moreover, if $f$ is concave and continuous (that is, continuous in 0 ) then the distorted probability $\mu \triangleq f \circ P$ is monotone, submodular and continuous from below (see Delbaen [8], [9], Denneberg [10], Kunze [23] for the proof, for further details and for applications to risk measures).

We recall (see Choquet [6] and Denneberg [10] for an exhaustive treatment) that for a normalized, monotone set function $\mu: \mathcal{F} \rightarrow[0,1]$ such that $\mu \ll P$ the Choquet integral of $X$, defined as

$$
E_{\mu}[X] \triangleq \int_{-\infty}^{0}[\mu(X \geq x)-1] d x+\int_{0}^{+\infty} \mu(X \geq x) d x
$$

satisfies the properties of monotonicity, positive homogeneity, comonotone additivity, cash additivity and $E_{\mu}\left[1_{A}\right]=\mu(A)$. Subadditivity holds iff $\mu$ is submodular. In particular, when $\mu$ is a probability measure then $E_{\mu}$ reduces to the classical expectation. 
Set

$$
\mathcal{M}(P) \triangleq\left\{\mu: \mathcal{F} \rightarrow[0 ;+\infty] \mid \begin{array}{c}
\mu \text { monotone set function such that } \\
\mu(\Omega)=1 \text { and } \mu \ll P
\end{array}\right\}
$$

It is clear that $\mathcal{M}(P)$ contains the set of all probability measures $Q \ll P$. In the following we will simply write $\mathcal{M}$ instead of $\mathcal{M}(P)$.

Song and Yan [25], [26] represented suitable functionals (without any axiom of continuity) in terms of set functions belonging to $\mathcal{M}$. More precisely, they proved that:

- if $\pi: L_{\mathcal{F}}^{\infty} \rightarrow \mathbb{R}$ satisfies monotonicity, comonotone subadditivity, positive homogeneity and cash additivity, then

$$
\pi(X)=\max _{\mu \in \mathcal{M}^{*}} E_{\mu}[X]
$$

where $\mathcal{M}^{*} \triangleq\left\{\mu \in \mathcal{M}: E_{\mu}[Y] \leq \pi(Y) \forall Y \in L_{\mathcal{F}}^{\infty}\right\}$;

- if $\pi: L_{\mathcal{F}}^{\infty} \rightarrow \mathbb{R}$ satisfies monotonicity, comonotone convexity and cash additivity, then

$$
\pi(X)=\max _{\mu \in \mathcal{M}}\left\{E_{\mu}[X]-F(\mu)\right\}
$$

where the penalty functional $F$ is defined as

$$
F(\mu) \triangleq \sup _{X \in L_{\mathcal{F}}^{\infty}: \pi(X) \leq 0} E_{\mu}[X]
$$

By imposing $\pi(0)=0$, it follows that $F(\mu) \geq 0$ and $\min _{\mu \in \mathcal{M}} F(\mu)=0$.

Remark 14 We show now that the following relation (proved by Föllmer and Schied [11], [12] in the convex case)

$$
F(\mu)=\sup _{X \in L_{\mathcal{F}}^{\infty}}\left\{E_{\mu}[X]-\pi(X)\right\}
$$

also holds for functionals satisfying comonotone convexity, monotonicity and cash additivity. that

By comonotone additivity of $E_{\mu}$ and by cash additivity of $\pi$ it follows indeed

$$
\begin{aligned}
\sup _{X \in L_{\mathcal{F}}^{\infty}}\left\{E_{\mu}[X]-\pi(X)\right\} & =\sup _{Y \in L_{\mathcal{F}}^{\infty}: Y=X-\pi(X)} E_{\mu}[Y] \\
& \leq \sup _{Y \in L_{\mathcal{F}}^{\infty}: \pi(Y) \leq 0}\left\{E_{\mu}[Y]-\pi(Y)\right\} \\
& \leq \sup _{X \in L_{\mathcal{F}}^{\infty}}\left\{E_{\mu}[X]-\pi(X)\right\}
\end{aligned}
$$

As a consequence of equation (15), the representation (12) can be rewritten as in $(13)$ with $F(\mu) \triangleq\left\{\begin{aligned} 0 & ; \quad \text { if } \mu \in \mathcal{M}^{*} \\ +\infty & ; \quad \text { otherwise }\end{aligned}\right.$. 


\subsection{On the dual representation of certain classes of risk measures}

Our aim is to represent functionals $\pi$ satisfying the properties above (comonotone convexity, monotonicity, cash additivity and $\pi(0)=0$ ) plus some kind of continuity. The assumption that $\pi(0)=0$ just implies that the penalty functional $F$ in the representation (13) is such that $\min _{\mu \in \mathcal{M}} F(\mu)=0$.

\section{Further axioms}

- continuity from above in 0 (shortly, ca0) of $\pi$ : for any sequence $\left(Y_{n}\right)_{n \in \mathbb{N}}$ such that $Y_{n} \searrow_{n} 0$, then $\lim _{n \rightarrow+\infty} \pi\left(Y_{n}\right)=\pi(0)=0$

- continuity from above in 0 of $\mu$ : for any sequence $\left(B_{n}\right)_{n \in \mathbb{N}}$ of sets such that $B_{n} \searrow_{n} \emptyset$, then $\lim _{n \rightarrow+\infty} \mu\left(B_{n}\right)=0$

By cash additivity of $\pi$ and $\pi(0)=0$, it is clear that continuity from above in 0 of $\pi$ is equivalent to the following axiom: for any sequence $\left(Y_{n}\right)_{n \in \mathbb{N}}$ such that $Y_{n} \searrow_{n} k$ with $k \in \mathbb{R}$, then $\lim _{n \rightarrow+\infty} \pi\left(Y_{n}\right)=k$.

In the following, $\mathcal{M}_{c a 0}$ will denote the subset of $\mathcal{M}$ formed by all normalized, monotone set functions $\mu \ll P$ that are continuous from above in 0 , i.e. $\mathcal{M}_{c a_{0}}=$ $\{\mu \in \mathcal{M}: \mu$ is ca0 $\}$.

The following result (and its proof) is an extension of Lemma 19 of Föllmer and Schied [12] to the comonotone case.

Proposition 15 Let $\pi: L_{\mathcal{F}}^{\infty} \rightarrow \mathbb{R}$ satisfy monotonicity, comonotone convexity, cash additivity and $\pi(0)=0$.

For any sequence $\left(Y_{n}\right)_{n \in \mathbb{N}}$ such that $0 \leq Y_{n} \leq 1$ for any $n \in \mathbb{N}$, the following are equivalent:

(a) $\pi\left(\lambda Y_{n}\right) \rightarrow_{n} 0$ for any $\lambda>0$;

(b) $\sup _{\mu \in \Lambda_{c}} E_{\mu}\left[Y_{n}\right] \rightarrow_{n} 0$ for any $c>0$,

where $\Lambda_{c} \triangleq\{\mu \in \mathcal{M}: F(\mu) \leq c\}$.

Proof. Consider an arbitrary sequence $\left(Y_{n}\right)_{n \in \mathbb{N}}$ such that $0 \leq Y_{n} \leq 1$ for any $n \in \mathbb{N}$.

(a) $\Rightarrow(b)$ : by the formulation of $F$ in (15), for any $\mu \in \Lambda_{c}$ (with fixed $c>0$ ) and for any $\lambda>0$ it holds

$$
c \geq F(\mu) \geq E_{\mu}\left[\lambda Y_{n}\right]-\pi\left(\lambda Y_{n}\right) .
$$

Hence:

$$
E_{\mu}\left[Y_{n}\right] \leq \frac{c}{\lambda}+\frac{\pi\left(\lambda Y_{n}\right)}{\lambda}
$$

By positivity of $Y_{n}$, we get

$$
0 \leq \sup _{\mu \in \Lambda_{c}} E_{\mu}\left[Y_{n}\right] \leq \frac{c}{\lambda}+\frac{\pi\left(\lambda Y_{n}\right)}{\lambda} .
$$


By (a), it follows therefore that

$$
0 \leq \varlimsup_{n} \sup _{\mu \in \Lambda_{c}} E_{\mu}\left[Y_{n}\right] \leq \lim _{n}\left(\frac{c}{\lambda}+\frac{\pi\left(\lambda Y_{n}\right)}{\lambda}\right)=\frac{c}{\lambda} .
$$

It is therefore sufficient to pass to the limit (as $\lambda \rightarrow+\infty$ ) to obtain (b).

(b) $\Rightarrow(a)$ : Again by positivity of $Y_{n}$ and by the representation of $\pi$ in (13):

$$
\begin{aligned}
0 \leq \pi\left(\lambda Y_{n}\right) & =\sup _{\mu \in \mathcal{M}}\left\{E_{\mu}\left[\lambda Y_{n}\right]-F(\mu)\right\} \\
& =\sup _{\mu \in \Lambda_{\lambda}}\left\{E_{\mu}\left[\lambda Y_{n}\right]-F(\mu)\right\} \\
& \leq \lambda \sup _{\mu \in \Lambda_{\lambda}} E_{\mu}\left[Y_{n}\right] \rightarrow_{n} 0
\end{aligned}
$$

where the limit in (17) is due to (b) and (16) is due to the fact that if $\mu \notin \Lambda_{\lambda}$ then $F(\mu)>\lambda$, hence $E_{\mu}\left[\lambda Y_{n}\right]-F(\mu)<E_{\mu}\left[\lambda Y_{n}\right]-\lambda \leq 0$.

$>$ From the inequalities above it follows $\pi\left(\lambda Y_{n}\right) \rightarrow_{n} 0$ for any $\lambda>0$.

The following result is an extension of Proposition 18 of Föllmer and Schied [12] to the comonotone case.

Proposition 16 Let $\pi: L_{\mathcal{F}}^{\infty} \rightarrow \mathbb{R}$ satisfy monotonicity, comonotone convexity, cash additivity and $\pi(0)=0$.

If $\pi$ is continuous from above in 0 , then:

$$
F(\mu)<+\infty \Rightarrow \mu \in \mathcal{M}_{c a 0} .
$$

Hence, $\pi$ can be represented as

$$
\pi(X)=\max _{\mu \in \mathcal{M}_{c a 0}}\left\{E_{\mu}[X]-F(\mu)\right\} .
$$

Proof. In order to prove that the set $\mathcal{M}$ can be relaxed with $\mathcal{M}_{c a 0}$, it is sufficient to consider an arbitrary sequence $\left(B_{n}\right)_{n \in \mathbb{N}}$ such that $B_{n} \searrow_{n} \emptyset$ and to take $Y_{n}=1_{B_{n}}$. In such a case, indeed, the sequence $\left(Y_{n}\right)_{n \in \mathbb{N}}$ satisfies the hypothesis of Proposition 15 and, because of the continuity from above in 0 of $\pi$, (a) is verified. Hence, $\sup _{\mu \in \Lambda_{c}} E_{\mu}\left[1_{B_{n}}\right] \rightarrow_{n} 0$ for any $c>0$. Therefore: if $F(\mu)<+\infty$ then there exists $c_{0}>0$ such that $F(\mu) \leq c_{0}$, hence $\mu \in \Lambda_{c_{0}}$ and $\mu\left(B_{n}\right) \rightarrow_{n} 0$, that is continuity from above of $\mu$ in 0 .

The last statement is immediate.

Corollary 17 Let $\pi: L_{\mathcal{F}}^{\infty} \rightarrow \mathbb{R}$ satisfy monotonicity, comonotone subadditivity, positive homogeneity and cash additivity.

If $\pi$ is continuous from above in 0 , then it can be represented as

$$
\pi(X)=\max _{\mu \in \mathcal{M}^{*}} E_{\mu}[X]
$$

for a suitable $\mathcal{M}^{*} \subseteq \mathcal{M}_{\text {ca0 }}$. 
Proof. Since comonotone subadditivity plus positive homogeneity imply comonotone convexity, by Proposition 16

$$
\pi(X)=\max _{\mu \in \mathcal{M}_{c a 0}}\left\{E_{\mu}[X]-F(\mu)\right\} .
$$

Consider now an arbitrary $\mu \in \mathcal{M}_{c a 0}$ and suppose that $F(\mu)>0$. Since $F(\mu)=\sup _{X \in L_{\mathcal{F}}^{\infty}}\left\{E_{\mu}[X]-\pi(X)\right\}$, we may suppose that there exists a $\bar{X} \in L_{\mathcal{F}}^{\infty}$ such that $E_{\mu}[\bar{X}]-\pi(\bar{X})>0$. Hence, by positive homogeneity of $\pi$,

$$
F(\mu) \geq \sup _{\lambda>0}\left\{E_{\mu}[\lambda \bar{X}]-\pi(\lambda \bar{X})\right\}=\sup _{\lambda>0}\left\{\lambda\left[E_{\mu}[\bar{X}]-\pi(\bar{X})\right]\right\}=+\infty
$$

The representation (19) then follows from the arguments above by taking $\mathcal{M}^{*}=$ $\left\{\mu \in \mathcal{M}_{c a 0}: F(\mu)<+\infty\right\}$.

\subsubsection{Law invariance and consistency with respect to different orders}

In this section we will assume that the probability space $(\Omega, \mathcal{F}, P)$ is atomless.

We recall the following definitions on different orders. For further details, see Dana [7], Kusuoka [24] and Song and Yan [26], among many others.

Definition 18 (see [7], [24], [26]) A random variable $X$ is said to be dominated by $Y$

- in the First Stochastic Dominance Order $\left(X \preceq_{1} Y\right)$ if $F_{X}(x) \geq F_{Y}(x)$ for any $x \in \mathbb{R}$

- in the Stop-Loss Order $\left(X \preceq_{s l} Y\right)$ if $E\left[(X-x)^{+}\right] \leq E\left[(Y-x)^{+}\right]$for any $x \in \mathbb{R}$

Definition 19 (see [7], [24], [26]) A functional $\pi$ is said to be consistent with the First Stochastic Dominance (resp. Stop-Loss) Order if:

$X \preceq_{1} Y\left(\right.$ resp. $\left.X \preceq_{s l} Y\right) \Rightarrow \pi(X) \leq \pi(Y)$

It is clear that consistency with the First Order Stochastic Dominance implies law invariance and monotonicity. The converse is also true in atomless spaces (see Kaas et al. [21] and Wang et al. [29], among many others).

Denote by $\mathcal{G}$ the set of all distortions, i.e. of all increasing functions $g$ : $[0,1] \rightarrow[0,1]$ satisfying $g(0)=0$ and $g(1)=1$, and by $\mathcal{G}^{c c}$ the set of all concave distortions.

We remind two results of Song and Yan [26]. The unique difference between the formulations below and those in [26] is that here $\pi(0)=0$ is imposed, hence $\min _{g \in \mathcal{G}} F(g \circ P)=0$.

Proposition 20 (Song and Yan; Theorem 3.5; [26]) $\pi: L^{\infty} \rightarrow \mathbb{R}$ satisfies consistency with respect to $\preceq_{1}$ (or, equivalently, law invariance and monotonicity), comonotone convexity, cash additivity and $\pi(0)=0$ if and only if

$$
\pi(X)=\max _{g \in \mathcal{G}}\left\{E_{(g \circ P)}[X]-F(g \circ P)\right\},
$$


where $F(g \circ P)=\sup _{X \in L_{\mathcal{F}}^{\infty}}\left\{E_{(g \circ P)}[X]-\pi(X)\right\}$ and $\min _{g \in \mathcal{G}} F(g \circ P)=0$.

A similar result holds also for the comonotone subadditive case (see [26]).

When continuity from above in 0 is imposed to $\pi$, we obtained the following particular case.

Proposition 21 (comonotone convexity)

Let $\pi: L_{\mathcal{F}}^{\infty} \rightarrow \mathbb{R}$ satisfy consistency with respect to $\preceq_{1}$ (or, equivalently, law invariance and monotonicity), comonotone convexity, cash additivity and $\pi(0)=0$.

If $\pi$ is continuous from above in 0 , then $\pi$ can be represented as in (21) by replacing $\mathcal{G}$ with

$$
\mathcal{G}_{\text {ca } 0} \triangleq\{g \in \mathcal{G}: g \text { is continuous in } 0\} \text {. }
$$

Proof. Since $g \circ P \in \mathcal{M}$ for any $g \in \mathcal{G}$, from Proposition 16 it follows that

$$
F(g \circ P)<+\infty \Rightarrow g \circ P \text { is ca0 } \Rightarrow g \text { is continuous in } 0,
$$

hence the thesis.

Proposition 22 (comonotone subadditivity and positive homogeneity)

Let $\pi: L_{\mathcal{F}}^{\infty} \rightarrow \mathbb{R}$ satisfy consistency with respect to $\preceq_{1}$ (or, equivalently, law invariance and monotonicity), comonotone subadditivity, positive homogeneity and cash additivity.

If $\pi$ is continuous from above in 0 , then

$$
\pi(X)=\max _{g \in \mathcal{G}_{c a 0}^{*}} E_{(g \circ P)}[X]
$$

for a suitable $\mathcal{G}_{c a 0}^{*} \subseteq \mathcal{G}_{c a 0}$.

Proof. Thanks to the result above, this proof can be driven as the proof of Corollary 17.

Proposition 23 (Song and Yan; Theorems 3.6 and 3.7; [26]) $\pi: L^{\infty} \rightarrow$ $\mathbb{R}$ satisfies cash additivity, comonotone convexity and consistency wrt $\preceq_{\text {sl }}$ iff $\pi$ satisfies cash additivity, convexity and consistency wrt $\preceq_{1}$.

Under the hypothesis above plus $\pi(0)=0$ :

$$
\pi(X)=\max _{g \in \mathcal{G}^{c c}}\left\{E_{(g \circ P)}[X]-F(g \circ P)\right\},
$$

where $F(g \circ P)=\sup _{X \in L_{\mathcal{F}}^{\infty}}\left\{E_{(g \circ P)}[X]-\pi(X)\right\}$ and $\min _{g \in \mathcal{G}^{c c}} F(g \circ P)=0$.

A similar result holds also for the comonotone subadditive case (see [26]). The representation (23) can be found also in Theorem 4.12 of Kunze [23] under the additional assumption of continuity from below of $\pi$ (already guaranteed by the other hypothesis - see Jouini et al. [20]).

By imposing continuity from above in 0 to $\pi$ and by applying Proposition 16 , we obtain the following particular cases. 
Corollary 24 (convexity) Let $\pi: L_{\mathcal{F}}^{\infty} \rightarrow \mathbb{R}$ satisfy law invariance, monotonicity, convexity, cash additivity (or, equivalently, consistency wrt $\preceq_{1}$, convexity and cash additivity; or, equivalently by Proposition 23, comonotone convexity, consistency wrt $\preceq_{\text {sl }}$ and cash additivity) and $\pi(0)=0$.

If $\pi$ is continuous from above in 0 , then $\pi$ can be represented as in (23) by replacing $\mathcal{G}^{c c}$ with

$$
\mathcal{G}_{c}^{c c} \triangleq\left\{g \in \mathcal{G}^{c c}: g \text { is continuous in }[0,1]\right\} .
$$

Proof. Since $g \circ P \in \mathcal{M}$ for any $g \in \mathcal{G}^{c c}$, it is straightforward to check (see the proofs above) that $g$ has to be continuous in 0 when $F(g \circ P)<+\infty$. Continuity in 0 together with concave distortion implies that $g$ has to be continuous in $[0,1]($ when $F(g \circ P)<+\infty)$.

Corollary 25 (subadditivity and positive homogeneity)

Let $\pi: L_{\mathcal{F}}^{\infty} \rightarrow \mathbb{R}$ satisfy law invariance, monotonicity, subadditivity, positive homogeneity and cash additivity (or, equivalently, consistency wrt $\preceq_{1}$, subadditivity, positive homogeneity and cash additivity; or, equivalently by [26], comonotone subadditivity, positive homogeneity, consistency wrt $\preceq_{\text {sl }}$ and cash additivity).

If $\pi$ is continuous from above in 0 (hence, see later, also continuous from above), then

$$
\pi(X)=\max _{g \in \mathcal{G}_{c}^{*, c c}} E_{(g \circ P)}[X]
$$

for a suitable $\mathcal{G}_{c}^{*, c c} \subseteq \mathcal{G}_{c}^{c c}$.

Take note that in the last two particular cases law invariance, monotonicity, convexity, cash additivity and $\pi(0)=0$ already guarantee that $\pi$ is continuous from below (see Theorem 1.2 of Jouini et al. [20]). Here we impose the additional property of continuity from above in 0 . See the next subsection for further details on the definition and on the comparison between different notions of continuity.

\subsection{Comparison between different kinds of continuity in the comonotone case}

Continuity from above of $\pi$ in 0 has been investigated previously. We consider now the following further notions of continuity for $\pi$ :

- continuity from above (shortly, ca): if $\left(X_{n}\right)_{n \geq 0} \subseteq L_{\mathcal{F}}^{\infty}, X_{n} \searrow_{n} X \in L_{\mathcal{F}}^{\infty}$, then $\lim _{n} \pi\left(X_{n}\right)=\pi(X)$

- continuity from below (shortly, cb): if $\left(Y_{n}\right)_{n \geq 0} \subseteq L_{\mathcal{F}}^{\infty}, Y_{n} \nearrow_{n} Y \in L_{\mathcal{F}}^{\infty}$, then $\lim _{n} \pi\left(Y_{n}\right)=\pi(Y)$

- continuity from below in 1 (shortly, cb1): for any sequence $\left(Y_{n}\right)_{n \in \mathbb{N}}$ such that $Y_{n} \nearrow_{n} 1$, then $\lim _{n \rightarrow+\infty} \pi\left(Y_{n}\right)=\pi(1)$. 
With continuity from below in 1 of $\mu$ we mean that for any sequence $\left(A_{n}\right)_{n \in \mathbb{N}}$ of sets such that $A_{n} \nearrow_{n} \Omega$, then $\lim _{n \rightarrow+\infty} \mu\left(A_{n}\right)=1$.

By cash additivity of $\pi$ and $\pi(0)=0$, it is clear that continuity from below in 1 of $\pi$ is equivalent to the following axiom: for any sequence $\left(Y_{n}\right)_{n \in \mathbb{N}}$ such that $Y_{n} \nearrow_{n} k$ with $k \in \mathbb{R}$, then $\lim _{n \rightarrow+\infty} \pi\left(Y_{n}\right)=k$.

We recall that $\pi: L_{\mathcal{F}}^{\infty} \rightarrow \mathbb{R}$ is said to be order lower semi-continuous with respect to the weak topology $\sigma\left(L_{\mathcal{F}}^{\infty}, L_{\mathcal{F}}^{1}\right)$ (or to satisfy the Fatou property) if for any uniformly bounded sequence $\left(X_{n}\right)_{n \geq 0}$ such that $X_{n} \stackrel{\text { a.s. }}{\rightarrow} X$ it holds $\pi(X) \leq \liminf _{n} \pi\left(X_{n}\right)$.

See Delbaen [8], Föllmer and Schied [11] and Biagini and Frittelli [4], among many others, for further details.

By translating well known results true for convex risk measures $\rho$ (see Delbaen [8], [9], Föllmer and Schied [11], [12], Klöppel and Schweizer [22] and Jouini et al. [20]) to $\pi(X) \triangleq \rho(-X)$, it follows that for $\pi: L_{\mathcal{F}}^{\infty} \rightarrow \mathbb{R}$ satisfying convexity, monotonicity, cash additivity and $\pi(0)=0$ the following implications are true:

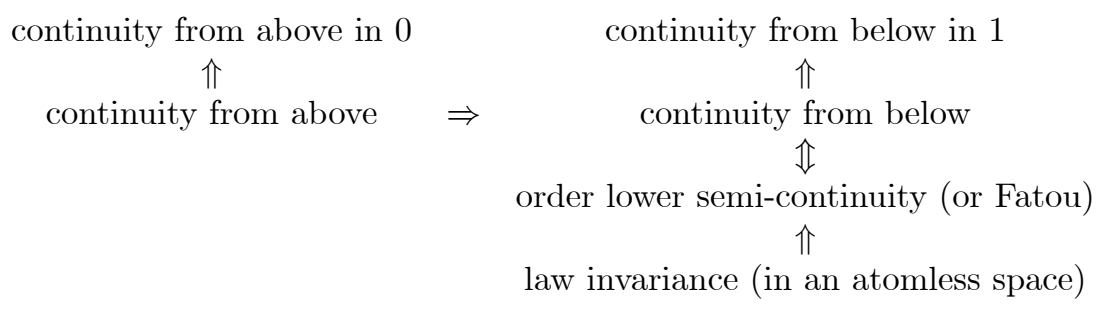

Furthermore, for $\pi$ satisfying also positive homogeneity: continuity from above is equivalent to continuity from above in 0 (see [8] and Section 3.2.1).

We investigate now if implications similar to the ones above hold also for comonotone convex $\pi$ and/or for continuity from above in 0 and from below in 1.

\subsubsection{Continuity from above (in 0 ) and continuity from below in 1}

We recall from Denneberg [10] that the conjugate set function $\bar{\mu}: \mathcal{F} \rightarrow[0,+\infty)$ of a normalized $\mu$ is defined as $\bar{\mu}(A) \triangleq 1-\mu\left(A^{c}\right)$, for any $A \in \mathcal{F}$, and that for a normalized monotone set function, continuity from above of $\mu$ is equivalent to continuity from below of $\bar{\mu}$ (see Proposition 2.3 of Denneberg [10]).

Lemma 26 Let $\mu$ be a normalized monotone set function such that $\mu \ll P$.

(i) $\mu$ is continuous from above in 0 iff $\bar{\mu}$ is continuous from below in 1 .

(ii) if $\mu$ is continuous from above in 0 (respectively, continuous from below in 1 ), then $E_{\mu}$ is continuous from above in 0 (respectively, continuous from below in 1). 
Proof. (i) Suppose that $\mu$ is continuous from above in 0 . Take an arbitrary sequence $\left(A_{n}\right)_{n \geq 0}$ such that $A_{n} \nearrow \Omega$. Then $\bar{\mu}\left(A_{n}\right)=1-\mu\left(A_{n}^{c}\right) \rightarrow_{n} 1$ since the sequence $\left(A_{n}^{c}\right)_{n \geq 0}$ satisfies $A_{n}^{c} \searrow \emptyset$.

The converse implication can be proved in a similar way.

(ii) can be checked easily as in the well known case of continuous from above/below $\mu$.

Lemma 27 Let $\pi$ satisfy convexity and $\pi(0)=0$, monotonicity and cash additivity. If $\pi$ is continuous from above in 0 , then it is also continuous from below in 1.

Proof. Suppose that $\pi$ is continuous from above in 0 and take an arbitrary sequence $\left(Y_{n}\right)_{n \in \mathbb{N}}$ such that $Y_{n} \nearrow 1$. Then $0 \geq \pi\left(Y_{n}\right)-1=\pi\left(Y_{n}-1\right)=$ $\pi\left(-\left(1-Y_{n}\right)\right) \geq-\pi\left(1-Y_{n}\right)$, where the last inequality is due to convexity and $\pi(0)=0$. From $1-Y_{n} \searrow 0$ and continuity from above of $\pi$ in 0 it follows that $\pi\left(1-Y_{n}\right) \rightarrow 0$, hence the thesis.

Under the additional hypothesis of positive homogeneity on $\pi$ a result similar to the previous one (formulated for risk measures) can be found in Delbaen [8].

It is easy to check that if $\pi$ is monotone, subadditive and continuous from above in 0 , then it is also continuous from above (see Delbaen [8]). Take indeed a sequence $\left(X_{n}\right)_{n \geq 0}$ such that $X_{n} \searrow_{n} X$. Then

$$
\pi(X) \leq \pi\left(X_{n}\right)=\pi\left(X_{n}-X+X\right) \leq \pi\left(X_{n}-X\right)+\pi(X) \rightarrow_{n} \pi(X),
$$

hence $\pi\left(X_{n}\right) \rightarrow_{n} \pi(X)$.

In the following example, we show that when convexity (or sublinearity) is weakened by comonotone convexity (in particular: comonotone additivity): (i) continuity from above in 0 does not imply continuity from below in 1; (ii) continuity from above in 0 does not imply continuity from above even if $\pi$ is monotone, comonotone subadditive and positively homogeneous; (iii) in an atomless space: law invariance is no more sufficient to guarantee continuity from below in 1 .

\section{Example 28 (ca0 and law invariant but neither cb1 nor ca) Consider}

$$
f(x)=\left\{\begin{array}{ll}
\frac{x^{2}}{2} ; & \text { if } 0 \leq x \leq \frac{1}{2} \\
\frac{x}{2} ; & \text { if } \frac{1}{2}<x<1 \\
1 ; & \text { if } x=1
\end{array} .\right.
$$

Take $([0,1), \mathcal{B}[0,1)$, Lebesgue $[0,1))$ as an atomless probability space and set $\pi(X)=E_{(f \circ P)}[X]$ for any $X \in L_{\mathcal{F}}^{\infty}$.

Hence $\mu=f \circ P$ is a monotone, normalized set function and $\mu \ll P$, so $\pi$ satisfies law invariance, monotonicity, positive homogeneity, comonotone additivity and cash additivity. Moreover, it is easy to check that continuity of $f$ in 0 implies continuity from above in 0 of $\mu$. Hence, by Lemma 26, $\pi$ is continuous from above in 0. 
- $\pi$ is not continuous from below in 1

For any $n \in \mathbb{N}, n \geq 2$, take now $A_{n}=\left[0 ; 1-\frac{1}{n}\right]$ and $Y_{n}=1_{A_{n}}$. Hence, $A_{n} \nearrow \Omega$ and $Y_{n} \nearrow 1$. It is immediate to check that $\pi(1)=1$. Nevertheless, for any $n>2$

$$
\pi\left(Y_{n}\right)=\int_{0}^{1} f\left(P\left(1_{A_{n}} \geq x\right)\right) d x=\int_{0}^{1} f\left(P\left(A_{n}\right)\right) d x=\frac{1}{2}-\frac{1}{2 n} \rightarrow_{n} \frac{1}{2} \neq \pi(1),
$$

i.e. $\pi$ is not continuous from below in 1 .

The motivation for which $\pi$ is continuous from above in 0 but not continuous from below in 1 is essentially that it is not subadditive. By taking $A=\left[0, \frac{1}{4}\right)$ and $B=\left[\frac{1}{2}, 1\right)$, it is easy to check that $\mu$ is not submodular and, therefore, $\pi$ is not subadditive.

- $\pi$ is not continuous from above

For any $n \in \mathbb{N}, n \geq 1$, take now $B_{n}=\left[0 ; \frac{1}{2}+\frac{1}{4 n}\right]$ and $X_{n}=1_{B_{n}}$. Hence, $B_{n} \searrow B=\left[0, \frac{1}{2}\right]$ and $X_{n} \searrow 1_{B}$. Nevertheless, for any $n \geq 1$

$$
\begin{aligned}
\pi\left(X_{n}\right) & =\int_{0}^{1} f\left(P\left(1_{B_{n}} \geq x\right)\right) d x=\int_{0}^{1} f\left(P\left(B_{n}\right)\right) d x=\frac{1}{4}+\frac{1}{8 n} \rightarrow_{n} \frac{1}{4} \\
\pi\left(1_{B}\right) & =\int_{0}^{1} f\left(P\left(1_{B} \geq x\right)\right) d x=\int_{0}^{1} f(P(B)) d x=\frac{1}{8}
\end{aligned}
$$

i.e. $\pi$ is not continuous from above. It is also clear that the distorted probability $\mu=f \circ P$ is continuous from above in 0 but not continuous from above.

The following counterexample is not surprising since $\operatorname{cb} 1 \nRightarrow$ ca0 even for $\pi$ induced by a coherent risk measure.

Example 29 (cb1 but not ca0) Take an increasing continuous function $f_{0}$ : $(0,1] \rightarrow(0,1]$ such that $f_{0}\left(0^{+}\right)=\lim _{x \backslash 0} f_{0}(x)>0$ and $f_{0}(1)=1$ and consider

$$
f(x)=\left\{\begin{array}{cl}
0 ; & \text { if } x=0 \\
f_{0}(x) ; & \text { if } 0<x \leq 1
\end{array}\right.
$$

Take $((0,1], \mathcal{B}(0,1]$, Lebesgue $(0,1])$ as a probability space and set $\pi(X)=$ $E_{(f \circ P)}[X]$ for any $X \in L_{\mathcal{F}}^{\infty}$.

As in Example 28, $\pi$ satisfies law invariance, monotonicity, positive homogeneity, comonotone additivity, cash additivity and $\pi(0)=0$. Moreover, it is easy to check that continuity of $f$ in 1 implies continuity from below in 1 of $\mu=f \circ P$. Hence, by Lemma 26, $\pi$ is continuous from below in 1 .

For any $n \in \mathbb{N}, n \geq 1$, take now $B_{n}=\left(0 ; \frac{1}{n}\right]$ and $X_{n}=1_{B_{n}}$. Hence, $B_{n} \searrow \emptyset$ and $X_{n} \searrow 0$. Nevertheless, for any $n \geq 1$

$\pi\left(X_{n}\right)=\int_{0}^{1} f\left(P\left(1_{B_{n}} \geq x\right)\right) d x=\int_{0}^{1} f\left(P\left(B_{n}\right)\right) d x=f_{0}\left(\frac{1}{n}\right) \rightarrow_{n} f_{0}\left(0^{+}\right)>\pi(0)$, i.e. $\pi$ is not continuous from above in 0 . 


\subsubsection{Order lower semi-continuity and continuity from below/above}

We focus now on the relationship between order lower semi-continuity (order lsc, for short) and continuity from below. It is well known that on $L_{\mathcal{F}}^{\infty}$ order lower semi-continuity and continuity from below are equivalent for functionals $\pi$ induced by convex risk measures (see Föllmer and Schied [13], among many others) and, more in general, for functionals $\pi$ satisfying monotonicity (see Lemma 15 of Biagini and Frittelli [4]).

In particular, by the arguments above for any $\pi: L_{\mathcal{F}}^{\infty} \rightarrow \mathbb{R}$ satisfying monotonicity:

order lsc $\Leftrightarrow$ continuous from below $\Rightarrow$ continuity from below in 1 .

The counterexample below shows that for $\pi$ satisfying monotonicity, comonotone convexity, cash additivity and $\pi(0)=0$ :

$$
\begin{array}{rll}
\text { continuity from below in } 1 & \nRightarrow & \text { order lsc } \\
\text { continuity from above } & \nRightarrow & \text { order lsc }
\end{array}
$$

even if we remind that the last implication is true at least when $\pi$ is convex.

It is also well known that (even for $\pi$ induced by coherent risk measures): $\mathrm{cb} \nRightarrow$ ca. Hence: order lsc $\nRightarrow$ ca.

Example 30 (cb1 and ca but not order lsc) Take $x_{0} \in(0,1)$, an increasing continuous function $f_{1}:\left[x_{0}, 1\right] \rightarrow(0,1]$ such that $f_{1}\left(x_{0}\right)>x_{0}$ and $f_{1}(1)=1$ and consider

$$
f(x)=\left\{\begin{array}{cl}
x ; & \text { if } 0 \leq x<x_{0} \\
f_{1}(x) ; & \text { if } x_{0} \leq x \leq 1
\end{array} .\right.
$$

Take $([0,1], \mathcal{B}[0,1]$, Lebesgue $[0,1])$ as a probability space and set $\pi(X)=$ $E_{(f \circ P)}[X]$ for any $X \in L_{\mathcal{F}}^{\infty}$.

As usual, $\pi$ satisfies law invariance, monotonicity, positive homogeneity, comonotone additivity, cash additivity and $\pi(0)=0$. Moreover, it is easy to check that $\pi$ is continuous from below in 1 and continuous from above.

For any $n \in \mathbb{N}, n \geq\left[1 / x_{0}\right]+1$, set $A_{n}=\left[0 ; x_{0}-\frac{1}{n}\right]$ and $Y_{n}=1_{A_{n}}$. Hence, $A_{n} \nearrow\left[0 ; x_{0}\right)=A$ and $Y_{n} \nearrow 1_{A} . \pi$ is not order lower semi-continuous, indeed for any $n \geq\left[1 / x_{0}\right]+1$

$$
\pi\left(Y_{n}\right)=\int_{0}^{1} f\left(P\left(A_{n}\right)\right) d x=\int_{0}^{1} f\left(x_{0}-\frac{1}{n}\right) d x=x_{0}-\frac{1}{n} \rightarrow_{n} x_{0}
$$

while

$$
\pi\left(1_{A}\right)=\int_{0}^{1} f(P(A)) d x=\int_{0}^{1} f\left(x_{0}\right) d x=f_{1}\left(x_{0}\right)>x_{0}=\liminf _{n} \pi\left(Y_{n}\right) .
$$

Example 28 (together with the results recalled above on order lsc) shows also that, for a monotone, cash additive, comonotone convex functional $\pi$ with $\pi(0)=0$ and in an atomless space, law invariance does not imply order lower semi-continuity. 


\subsection{A sufficient condition for continuity}

Let $\mathcal{X}$ be a Frechet lattice. Biagini and Frittelli [4] proved that any proper convex and monotone $\pi: \mathcal{X} \rightarrow(-\infty,+\infty)$ is continuous on the interior of the domain of $\pi$ (Extended Namioka-Klee Theorem - Theorem 1 in [4]). It is easy to check (see Lemma 1, [4]) that if $\pi: \mathcal{X} \rightarrow(-\infty,+\infty]$ is convex and satisfies $\pi(0)=0$ then

$$
n|\pi(X)| \leq \pi(n|X|), \quad \forall n \geq 1, \forall X \in \mathcal{X}
$$

Notice that (26) alone implies $\pi(0)=0$. Moreover, we observe that, in the proof of the mentioned Extended Namioka-Klee Theorem, only the properties

$$
|\pi(X)| \leq \frac{1}{n} \pi(n|X|), \quad \forall X \in \mathcal{X} \text { and for all large } n
$$

and monotonicity are used to prove the continuity in 0 of $\pi$. As stated before, (27) is satisfied by convex maps null in 0 , but may be satisfied by functional that are not convex. We therefore obtain a sufficient criterium for continuity:

Lemma 31 Let $\mathcal{X}$ be a Frechet lattice and $\pi: \mathcal{X} \rightarrow \mathbb{R}$ be a monotone real-valued functional. If $\pi$ satisfies (27) then it is continuous in 0 .

As the following example illustrates, monotone and quasiconvex functional $\pi: L_{\mathcal{F}}^{\infty} \rightarrow \mathbb{R}$ may not be continuous in 0 .

Example 32 Let $\mathcal{X}=\left(L_{\mathcal{F}}^{\infty},\|\cdot\|_{\infty}\right)$ and consider a strictly increasing function $\nu: \mathbb{R} \rightarrow \mathbb{R}$ satisfying $\nu(0)=0$. Then the functional $\pi: L_{\mathcal{F}}^{\infty} \rightarrow \mathbb{R}$

$$
\pi(X) \triangleq \nu(E[X])
$$

is increasing, satisfies $\pi(0)=0$ and is quasiconvex. In this case, (27) reduces to:

$$
|\nu(E[X])| \leq \frac{1}{n} \nu(n E[|X|]), \quad \forall X \in \mathcal{X} \text { and for all large } n \text {. }
$$

If $\nu$ does not grow too fast (i.e. if $\frac{\nu(|x|)}{|x|} \leq k \in \mathbb{R}_{+}$for large $x$ ) then $0 \leq$ $\frac{1}{n} \nu(n E[|X|]) \leq k E[|X|]$ and $\frac{1}{n} \nu(n E[|X|])$ is small if $E[|X|]$ is small. On the other hand, if $\nu$ is left discontinuous at 0 (i.e. $\nu\left(0^{-}\right) \triangleq \lim _{x \uparrow 0} \nu(x)<\nu(0)=0$ ), then $|\nu(E[X])| \geq\left|\nu\left(0^{-}\right)\right|$for all $E[X]<0$ and therefore property (28) is not satisfied. Clearly the discontinuity of $\nu$ implies that $\pi$ is not norm continuous in 0 .

\section{References}

[1] C.D. Aliprantis and K.C. Border, Infinite Dimensional Analysis, Springer, Second edition (1999).

[2] F. Bellini and M. Frittelli, Certainty equivalent and no arbitrage: a reconciliation via duality theory, Technical Report \# 139, Dept. "Metodi Quantitativi", University of Brescia (1998). 
[3] F. Bellini and M. Frittelli, On the existence of minimax martingale measures, Mathematical Finance 12 (2002) 1-21.

[4] S. Biagini and M. Frittelli, On the extension of the Namioka-Klee theorem and on the Fatou property for risk measures, in: Optimality and risk: modern trends in mathematical finance, The Kabanov Festschrift, F. Delbaen, M. Rasonyi, Ch. Stricker eds. (2009),1-28.

[5] S. Cerreia-Vioglio, F. Maccheroni, M. Marinacci and L. Montrucchio, Risk measures: rationality and diversification, The Carlo Alberto Working Paper no. 100 (2009).

[6] G. Choquet, Theory of capacities, Annales de l'Institut Fourier (Grenoble) 5 (1953) 131-295.

[7] R.A. Dana, A representation result for concave Schur concave functions, Mathematical Finance 15 (2005) 613-634.

[8] F. Delbaen, Coherent Risk Measures, Lecture notes, Scuola Normale Superiore, Pisa, Italy (2000).

[9] F. Delbaen, Coherent Risk Measures on General Probability Spaces, in: Advances in Finance and Stochastics, K. Sandmann and P.J. Schönbucher eds. (2002), Springer-Verlag, 1-37.

[10] D. Denneberg, Non-additive measure and integral, Kluwer Academic Publishers (1994).

[11] H. Föllmer and A. Schied, Convex measures of risk and trading constraints, Finance 8 Stochastics 6 (2002a) 429-447.

[12] H. Föllmer and A. Schied, Robust preferences and convex measures of risk, in: Advances in Finance and Stochastics, K. Sandmann and P.J. Schönbucher eds. (2002b), Springer-Verlag, 39-56.

[13] H. Föllmer and A. Schied, Stochastic Finance. An Introduction in Discrete Time, De Gruyter, Berlin, New York, Second edition (2004).

[14] M. Frittelli, The Minimal Entropy Martingale Measure and the Valuation Problem in Incomplete Markets, Mathematical Finance 10 (2000) 39-52.

[15] M. Frittelli, Representing sublinear risk measures and pricing rules, Working paper n. 10 (2000), Università di Milano Bicocca, Italy.

[16] M. Frittelli and M. Maggis, Dual representation of quasiconvex conditional maps, Preprint (2009): arXiv:1001.3644.

[17] M. Frittelli and M. Maggis, Conditional Certainty Equivalent, Preprint (2010). 
[18] M. Frittelli and E. Rosazza Gianin, Putting order in risk measures, Journal of Banking \&3 Finance 26 (2002) 1473-1486.

[19] C.C. Heyde, S.G. Kou and X.H. Peng, What is a good risk measure: bridging the gaps between data, coherent risk measure and insurance risk measure, Preprint (2006).

[20] E. Jouini, W. Schachermayer and N. Touzi, Law Invariant Risk Measures have the Fatou Property, in: Kusuoka, S., Yamazaki, A. (Eds.), Advances in Mathematical Economics, vol. 9 (2006), pp. 49-71.

[21] R. Kaas, A.E. van Heerwaaden and M.J. Goovaerts, Ordering of Actuarial Risks, Education Series 1, CAIRE, Brussels (1994).

[22] S. Klöppel and M. Schweizer, Dynamic utility indifference valuation via convex risk measures, Working Paper FINRISK no. 209 (2005), ETH Zürich.

[23] M. Kunze, Verteilungsinvariante konvexe Risikomaße, Diplomarbeit, Humboldt-Universität, Berlin (2003).

[24] S. Kusuoka, On law invariant coherent risk measures. In: Kusuoka, S., Maruyama, T. (eds.): Advances in Mathematical Economics, Vol. 3 (2001), pp. 83-95.

[25] Y. Song and J.-A. Yan, The representations of two types of functionals on $L^{\infty}(\Omega, \mathcal{F})$ and $L^{\infty}(\Omega, \mathcal{F}, P)$, Science in China: Series A Mathematics 49 (2006) 1376-1382.

[26] Y. Song and J.-A. Yan, Risk Measures with Comonotonic Subaddivity or Convexity and Respecting Stochastic Orders, Insurance: Mathematics and Economics 45 (2009) 459-465.

[27] Y. Song and J.-A. Yan, An overview of representation theorems for static risk measures, Science in China: Series A Mathematics 52 (2009) 14121422 .

[28] M. Volle, Duality for the level sum of quasiconvex functions and applications, Control, Optimisation and Calculus of variations 3 (1998) 329-343.

[29] S.S. Wang, V.R. Young and H.H. Panjer, Axiomatic characterization of insurance prices, Insurance: Mathematics and Economics 21 (1997) 173183. 